\title{
A Neural Network Approach to Predicting Car Tyre Micro-Scale and Macro-Scale Behaviour
}

\author{
Xiaoguang Yang, Mohammad Behroozi, Oluremi A. Olatunbosun
}

School of Mechanical Engineering, University of Birmingham, Birmingham, UK.

Email: o.a.olatunbosun@bham.ac.uk

Received October $2^{\text {nd }}, 2013$; revised November $2^{\text {nd }}, 2013$; accepted November $9^{\text {th }}, 2013$

Copyright (C 2014 Xiaoguang Yang et al. This is an open access article distributed under the Creative Commons Attribution License, which permits unrestricted use, distribution, and reproduction in any medium, provided the original work is properly cited. In accordance of the Creative Commons Attribution License all Copyrights @ 2014 are reserved for SCIRP and the owner of the intellectual property Xiaoguang Yang et al. All Copyright (c) 2014 are guarded by law and by SCIRP as a guardian.

\section{ABSTRACT}

Finite Element (FE) analysis has become the favoured tool in the tyre industry for virtual development of tyres because of the ability to represent the detailed lay-up of the tyre carcass. However, application of FE analysis in tyre design and development is still very time-consuming and expensive. Here, the application of various Artificial Neural Network (ANN) architectures to predicting tyre performance is assessed to select the most effective and efficient architecture, to allow extensive parametric studies to be carried out inexpensively and to optimise tyre design before a much more expensive full $\mathrm{FE}$ analysis is used to confirm the predicted performance.

\section{KEYWORDS}

\section{Design Parameters; Finite Element Modelling; Neural Network; Tyre Configuration}

\section{Introduction}

The vehicle tyre, in its interaction with the road, generates the forces and moments which control vehicle motion. Of particular importance are the tyre radial force which controls vehicles ride behaviour, longitudinal force which controls acceleration/braking and lateral force which controls cornering and directional stability. These forces are functions of tyre dynamic characteristics and result from deformation of the tyre due to tyre/road interaction. Static stiffness, determined by measuring its elastic deformation and contact patch information is one of the important mechanical properties of a tyre [1]. Since this and other performance characteristics are dependent on the detailed design configuration of the tyre, there is increasing use of virtual prototyping tools such as finite element (FE) analysis in the design and development of new tyre products to evaluate different tyre design configurations.

Kazuyuki Kabe and Masataka Koishi [2] investigated tyre cornering behaviour by both implicit and explicit finite element analysis considering the modelling details of tyre composite structure and nonlinear material properties. The simulation results were verified using a flat- trac tyre test system. O. A. Olatunbosun and A. M. Burke studied the tyre dynamic behaviour in the time domain by finite element analysis for better understanding of tyre ride response [3]. P. Ghosh et al. carried out a study on the effect of tyre design parameters on tyre performance for optimizing tyre configuration [4]. From the aforementioned studies on finite element tyre modelling, it has been shown that a finite element tyre model can provide data, not only for comparison to measured test data, but also data that are impractical to measure, and are thus an efficient alternative to the conventional design, build and test method of developing tyre prototypes.

Further efficiencies can be gained in the development of tyre prototypes by reducing the computational cost of finite element analysis through the application of artificial intelligence technology such as artificial neural network (ANN). The ANN based method has been used widely in different research fields in the past decades, such as modelling complex systems [5], fault detection and diagnosis [6] and sales forecasting [7], etc. The use of artificial intelligence based methods in tyre design is a recent development. For example, a fuzzy logic based method was developed and applied for evaluating tyre 
dynamic parameters such as tyre contact area, cornering stiffness, and tyre-road friction coefficient for application in vehicle control systems [8]. ANN based methods have also been proposed for estimating tyre/road friction force [9] for predicting tyre handling performance [10], and for modelling tyre failure rate [11].

Olatunbosun et al. [12] investigated the application of a tyre virtual prototyping environment, combining FE analysis and ANN technology, to investigate the effect of tyre belt ply design configuration parameters on tyre static stiffness and tyre/road contact characteristics. Such an ANN model can be employed in the initial evaluation and rapid optimisation of new tyre designs before more expensive FE analysis is used to confirm the predicted performance.

The current paper gives a detailed exposition of the development of the ANN model, with consideration of four different ANN structures and comparison of their performance in terms of accuracy and cost. The ANN model was trained using test data generated from a validated finite element tyre model developed using the commercial finite element software ABAQUS ${ }^{\mathrm{TM}}$. This allowed the tyre belt reinforcement cords to be modelled in detail. Using different tyre belt design configurations, a large number of different tyre designs were created and their performance characteristics obtained by FE simulation. The tyre design parameters were the input data and the tyre performance parameters obtained from the virtual tests were the output data used to train the ANN tyre model. Once trained, the ANN model would provide a reliable, fast and efficient tool for predicting the performance characteristics of new tyre designs with different belt configurations without having to use FE analysis.

Such an ANN model can be employed in the initial evaluation and rapid optimisation of new tyre designs before more expensive $\mathrm{FE}$ analysis is used to confirm the predicted performance.

\section{Tyre Material Properties}

As a composite structure, tyres have complex characteristics due to their diverse material properties and complex structure. Generally, the tyre is constructed using high modulus cord and low modulus rubber. Several layers of cord spaced in the rubber act as the reinforcing frame of the tyre. The reinforcement layers enveloped by the rubber exhibit highly nonlinear stress-strain behaviour.

This study is based on a slick radial tyre 175/505 R13 for SAE Formula Student (FS). Currently, over one hundred FS racing teams are using different makes of tyre, such as Dunlop, Goodyear, and Hoosier. These tyres usually work under relatively low pressure and load. The growing popularity of FS makes the competition keener and has forced the FS teams to strive for higher performance of their cars. As a cornerstone of FS racing car design, the tyres' characteristics are now being paid more attention and have resulted in taking up to two seconds off the lap time during competition [13].

The tyre consists of different rubber components with embedded reinforcements. The dominant component of the tyre is rubber including tread, under-tread, sidewall, apex, and the inner-liner. The reinforcement of the tyre includes the belt, carcass, bead, cap ply, and bead reinforcement. The rubber material properties for different components such as tread and sidewall are not identical due to the different mixed proportions of rubber and carbon black [14]. The function of tread rubber is to keep the balance of tyre performance and durability, while the sidewall rubber is designed mainly for fatigue resistance.

The availability of accurate knowledge of a tyre's constituent material properties is vital for constructing a FE model capable of representing the behaviour of the tyre accurately. However, tyre material properties are usually not available to most researchers due to the proprietary protection from the tyre manufacturers. The material properties for the tyre were determined using techniques developed by Yang et al. [15].

Based on a series of uni-axial tests and evaluations using ABAQUS, the Yeoh hyperelastic model constants for different rubber components were obtained and listed in Table 1.

In ABAQUS, the viscoelastic material property should be considered together with the hyperelastic model to define large-deformation, nonlinear, viscoelastic behaviour. Likewise, the viscoelastic material model must be combined with the isotropic linear elasticity model to define classical, linear, small-strain, viscoelastic behaviour. The normalised relaxation test data (Table 2) were utilised to evaluate the Prony series parameters in ABAQUS as described in [15].

Table 1. Hyperelastic Material Constants [15].

\begin{tabular}{cccc}
\hline Rubber Material & Yeoh strain energy potentials constants \\
\hline Component & $\mathrm{C} 10\left(\mathrm{~N} / \mathrm{mm}^{2}\right)$ & $\mathrm{C} 20\left(\mathrm{~N} / \mathrm{mm}^{2}\right)$ & $\mathrm{C} 30\left(\mathrm{~N} / \mathrm{mm}^{2}\right)$ \\
\hline Tread & 0.1714 & $-4.4041 \mathrm{E}-02$ & $1.7383 \mathrm{E}-2$ \\
Sidewall & 0.2933 & $-8.6149 \mathrm{E}-02$ & $3.7144 \mathrm{E}-02$ \\
Apex & 1.7245 & -2.2922 & 5.0544 \\
Inner-liner & 0.3223 & $-8.6751 \mathrm{E}-02$ & $2.4762 \mathrm{E}-02$ \\
Toeguard & 3.3175 & 4.7823 & 63.5242 \\
\hline
\end{tabular}


Table 2. Viscoelastic material constants [15].

\begin{tabular}{ccccc}
\hline Rubber Material & \multicolumn{2}{c}{ Relaxation Modulus $(\mathrm{g})$} & \multicolumn{2}{c}{ Relaxation Time $(\tau)$, sec. } \\
\hline Component & $g_{1}$ & $g_{2}$ & $\tau_{1}$ & $\tau_{2}$ \\
\hline Tread & 0.1433 & 0.0852 & 7.6125 & 235.62 \\
Sidewall & 0.1613 & 0.0773 & 6.2257 & 224.29 \\
Inner-liner & 0.1017 & 0.0839 & 19.6480 & 363.70 \\
Apex & 0.1815 & 0.1197 & $3.5618 \mathrm{E}-02$ & 206.28 \\
Toeguard & 0.1171 & 0.0891 & 21.2580 & 350.13 \\
\hline
\end{tabular}

In ABAQUS, the reinforcements are modelled using rebar elements embedded into the rubber matrix. In this way, the reinforcement component and the host component (rubber) are described in different constitutive models. The material properties can be assigned to their corresponding component, separately.

Since the deformation of cords/steel wire during tyre inflation and deflection produces significantly smaller strains compared to rubbers, the material properties of cords remain well within their linear elastic limits hence their non-linear material properties are not required. Thus, the Young's modulus $\mathrm{E}$ and the Poisson's ratio $v$ were used to define the material property of reinforcement cords.

\section{Finite Element Modelling}

The pneumatic tyre has a complex structure consisting of reinforcement plies and rubber components. In order to achieve successful prediction of tyre characteristics using FEA, an accurate 3D tyre model is crucial. Apart from the tyre material properties, the fundamental requirement for the tyre modelling process is precise and adequate information about tyre geometry.

Finite element analysis is now widely used as a powerful virtual simulation tool for tyre development because of its ability to model the detailed structure of a pneumatic tyre. Here, the tyre FE model used in the simulation experiment was developed in the commercial FE package ABAQUS to acquire data for training the ANN model.

Once the tyre cross-section structure details were obtained, the finite element model of cord-rubber composite was created in ABAQUS. In this study, the rebar layer function in ABAQUS provides the support for modelling cord as rebar element, which is embedded in rubber solid element.

The rebar layer function in ABAQUS is used to define the cord orientation, spacing, thickness, location, and material properties that are independent of rubber material properties definition. Material properties of different components obtained from tests were then applied to the model. The modelling procedure introduced in [14] was adopted in this study. A 2D axisymmetric tyre model was developed firstly by importing the $2 \mathrm{D}$ cross-section profile shown in Figure 1 into ABAQUS/CAE. The hybrid

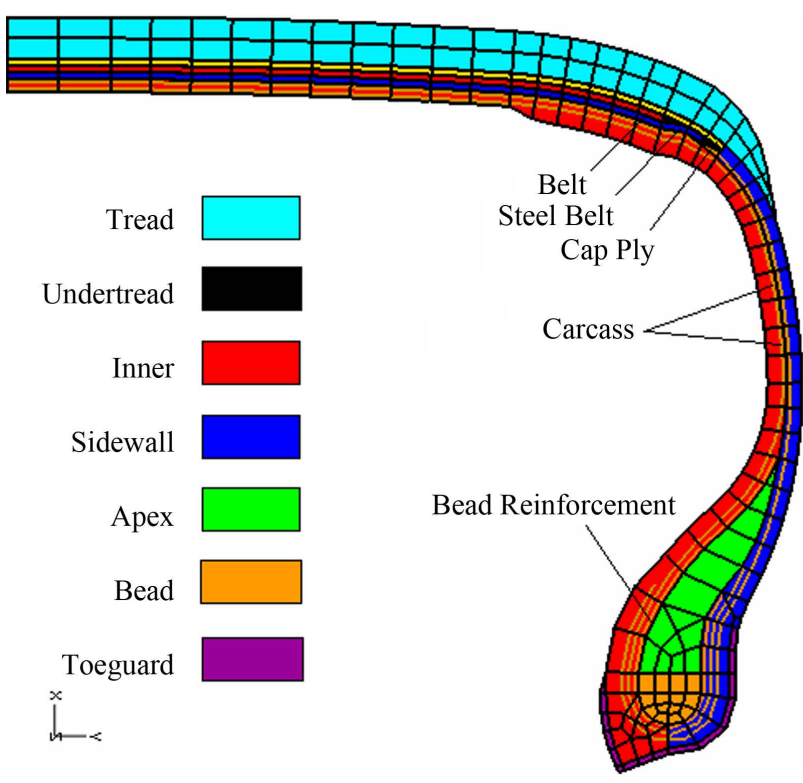

Figure 1. Finite element model of tyre cross-section in ABAQUS.

axisymmetric continuous elements with twist degree of freedom (CGAX4H and CGAX3H) were used to account for the twist feature of the cord along the axisymmetric axis due to anisotropy of fibre reinforcements [2]. Particularly, for the bead region, 2D axisymmetric hybrid element CGAX4H was used with steel property rather than embedding steel wire in rubber, which is effective and efficient. The reinforcement components were represented by the SFMGAX1 elements with 2 nodes, which were embedded in rubber elements in ABAQUS. One layer cap ply with 0 degree orientation (along the circumferential direction), two layers breakers including one layer steel belt with 20 degree orientation and one layer nylon belt with -20 degree orientation, and two layers carcass with 90 degree orientation were modelled by rebar layer and embedded in the corresponding host solid elements. The element set and node set were created for different components for convenient operation in ABAQUS, such as assigning material properties and boundary conditions, and post-processing.

The 2D axisymmetric model was converted into a 3D finite element tyre model using the symmetric model generation function in ABAQUS. During this process, 
the 2D axisymmetric elements (CGAX4H and CGAX3H) were transformed into 3D solid elements (C3D8H and C3D6H). The number of circumferential sections can be controlled for different analyses; for example, only the contact patch part of the tyre has refined mesh for steady state tyre rolling analysis. In this case, $50 \times 6.0^{\circ}$ circumferential coarse sections and $20 \times 3.0^{\circ}$ refined sections for the contact patch respectively were created.

This non-uniform mesh is appropriate not only to ensure accurate prediction but also low cost of computational time. The structure details such as the cord-rubber composite are still kept after revolving the 3D model.

\section{Virtual Tests on Pneumatic Tyre}

A number of virtual tests were carried out on the 3D FE tyre model including static load-deflection tests in the vertical, lateral, and longitudinal directions as well as tyre footprint analysis. These were then validated with tests on the tyre carried out on the University of Birmingham tri-axial electro-hydraulic tyre dynamic rig.

Results of the vertical stiffness validation are shown in Table 3 with tyre vertical stiffness determined at normal inflation pressure $80 \mathrm{kPa}$ as well as at $\pm 25 \%$ and $\pm 50 \%$ for the tyre in its normal design configuration.

Figure 2 shows footprint shape comparison between experiment and finite element analysis at normal inflation pressure $80 \mathrm{kPa}$ and normal vertical load $750 \mathrm{~N}$. The variations of tyre footprint area, due to the variation of inflation pressure and vertical load for both virtual and physical experiments, are shown in Figures 3 and 4.

It is evident that the tyre finite element model is capable of predicting the variations of the footprint area due to the changes of inflation pressure and vertical load. The trend of tyre contact area considering the variation of tyre inflation pressure and vertical load are consistent with the findings in [16] i.e. tyre footprint area increasing with increasing vertical load and decreasing inflation pressure.

The validation results for the finite element model show that it is capable of accurately predicting tyre performance characteristics. It therefore provides a reliable platform for investigating the tyre stiffness property.

\section{Methodology and Implementation}

\subsection{Artificial Neural Networks (ANN)}

A detailed description of artificial neural networks and the principles of neural modelling is given in [12]. Further details of the operational principles and the design of artificial neural networks can be found in standard textbooks e.g. Samarasinghe [17]. On the other hand, another important property of a neural network is its ability to learn complex nonlinear relationships between the inputs and outputs of the network [12]. This study takes advantage of this property of a neural network and its ability to improve its performance through learning, just like the human brain. Here, the application of various ANN architectures as the approximation tool for predicting tyre performance is assessed, including Multi-Layer Feedforward Networks or Multi-Layer Perceptron (MLP), Co-Active Neuro-Fuzzy system (CANFIS), Radial-based Function (RBF) neural network, and generalised feedforward MLP. A brief introduction of each network is presented as follows.

Multi-Layer Perceptron (MLP) Neural Network: In the multilayer networks, there are one or more hidden layers, whose nodes are correspondingly called hidden neurons. The back-propagation learning algorithm network typically trains the network by employing the deviation of outputs from corresponding desired values to correct and update the weights of the previous layer [17].

Co-Active Neuro-Fuzzy Interface system (CANFIS): The CANFIS model combines fuzzy logic input with a modular neural network to estimate complex functions for obtaining higher accuracy along with lower calculation cost due to its reduced structure. In addition, human knowledge is not required since the CANFIS model learns training data set by optimising the fuzzy membership function parameters with either error back-propagation or genetic algorithm (GA) [18].

Radial-basis Function (RBF) Neural Network: Radial basis function (RBF) networks are nonlinear structures, similar to MLPs, but constructed of a single hidden layer. Gaussian transfer function is used in RBF networks instead of the standard functions employed by MLPs. Since the learning in hidden layer is substituted by unsupervised learning, the learning cost and problem size is smaller, and as a result, network training will be faster [19].

Generalised feed-forward MLP (GFMLP) Neural Network: Generalised feed-forward networks are a generalisation of the MLP such that connections can jump over one or more layers [20]. The efficiency of this network is

Table 3. Vertical stiffness validation.

\begin{tabular}{cccc}
\hline Inflation Pressure $(\mathrm{kPa})$ & Vertical Stiffness Test $(\mathrm{N} / \mathrm{mm})$ & Vertical Stiffness Simulation $(\mathrm{N} / \mathrm{mm})$ & Absolute Difference $(\%)$ \\
\hline 40 & 51.181 & 51.439 & 0.50 \\
60 & 68.898 & 67.419 & 2.15 \\
80 & 84.786 & 82.813 & 2.33 \\
100 & 96.457 & 97.249 & 0.82 \\
120 & 112.02 & 112.34 & 0.29 \\
\hline
\end{tabular}




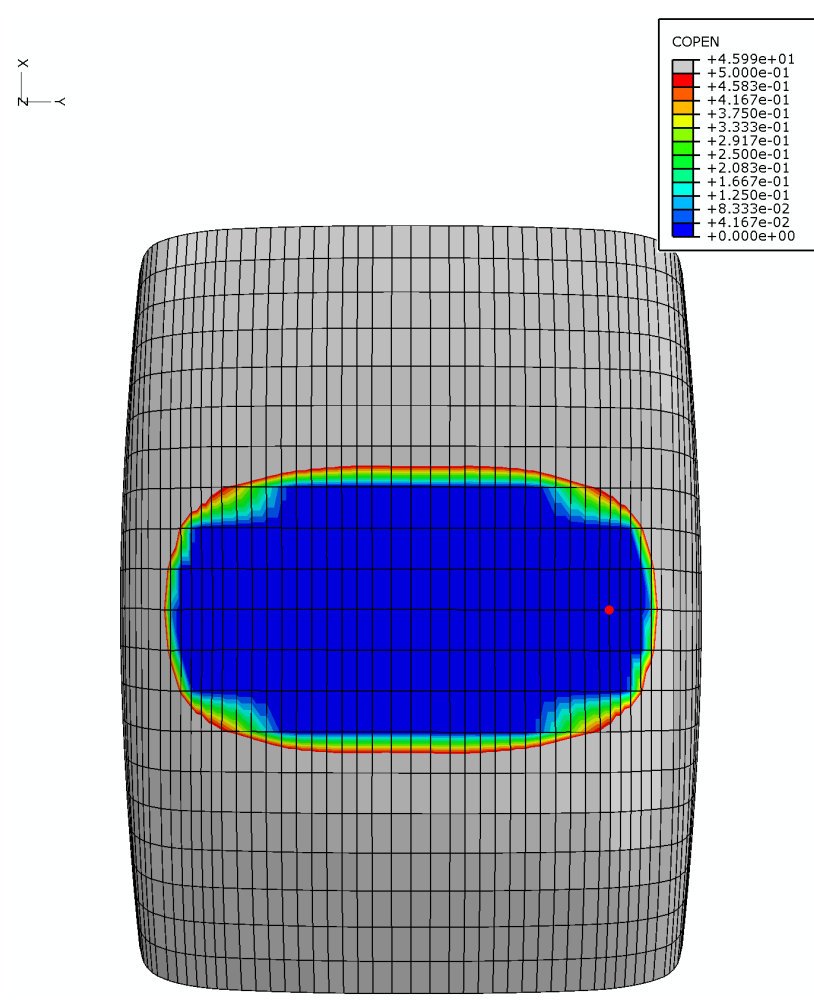

(a) Footprint from “COPEN"

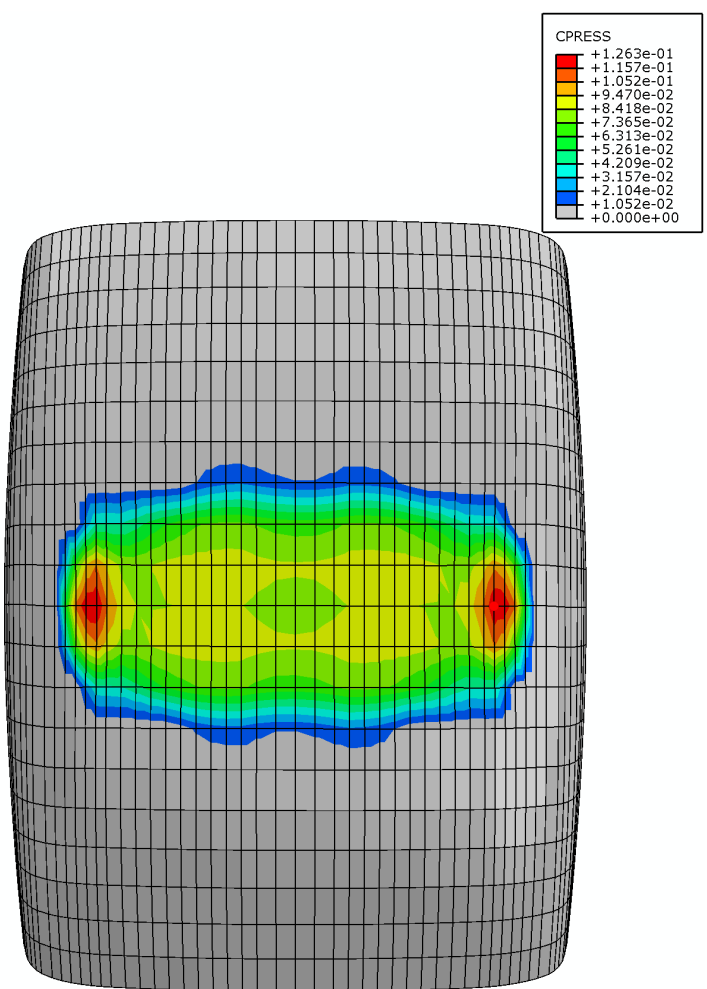

(b) Footprint from “CPRESS”

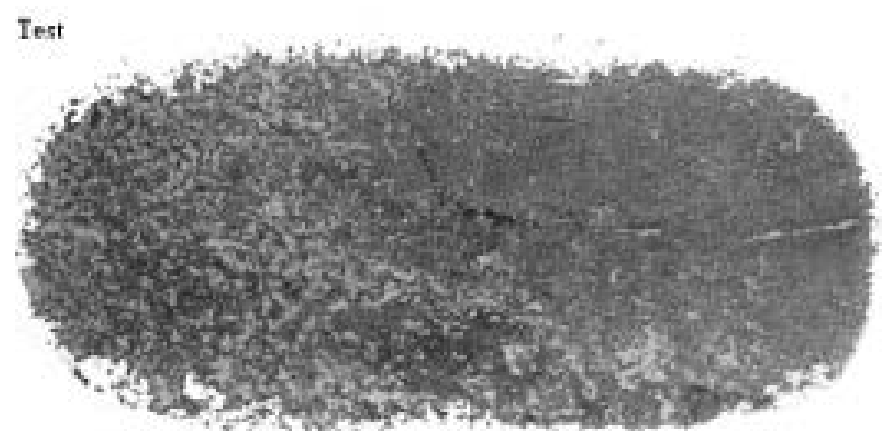

(c) Footprint from Test

Figure 2. Footprint shape comparison at inflation pressure $80 \mathrm{kPa}$ and vertical load $750 \mathrm{~N}$.

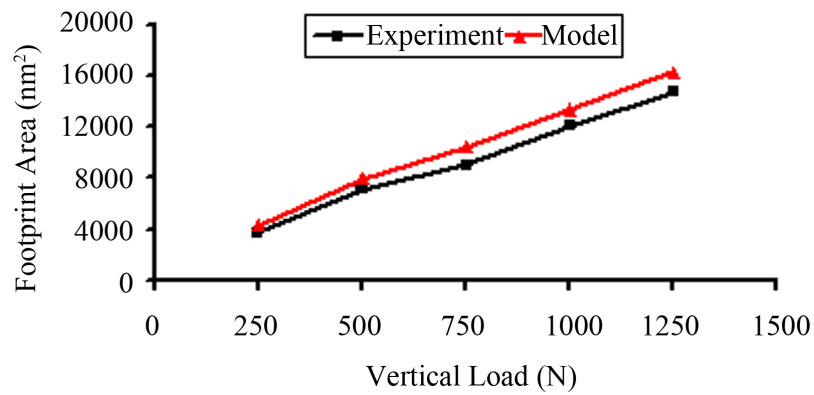

Figure 3. Variation of footprint area with respect to vertical load at $80 \mathrm{kPa}$ inflation pressure.

effectively higher than MLP since the network flexibility, adaptability, and nonlinearity is sensibly stronger than in normal MLPs.

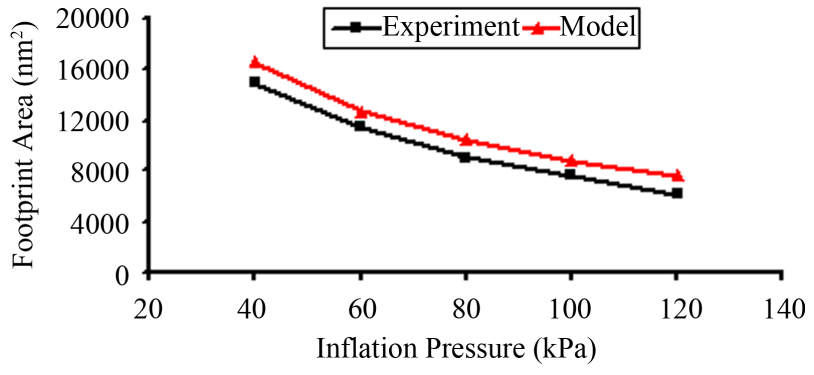

Figure 4. Variation of footprint area with respect to inflation pressure at $750 \mathrm{~N}$ vertical load.

\subsection{Data Acquisition}

Any artificial neural network requires to be trained with adequate data if it is to provide accurate prediction of the 
desired outputs from the given inputs. In this case, the inputs are the tyre belt ply design configuration parameters namely, cord orientation angle, spacing (cords end density), cross-sectional area, material property (Young's modulus). In addition, the operational condition, inflation pressure was considered.

The desired outputs are the tyre static stiffness property (in the radial, longitudinal and lateral directions), contact patch area, contact pressure and shear stress in the belt plane. To provide the training data, the independent input parameters were varied as shown in Table 4 to create 243 samples (prototype tyres). ABAQUS input decks were generated for each sample. Each input parameter was given a change of $\pm 10 \%$ apart from inflation pressure which was given a change of $\pm 25 \%$ because of the lower sensitivity of output parameters to it.

In the previous study [12], it was established that ANN technology could be applied to tyre design. Here the aim is to explore the efficiency and effectiveness of different ANN architectures in predicting tyre performance. This is achieved by comparing the tyre performance parameters predicted by the trained neural networks of different architectures to those predicted by a validated finite element model.

The chosen outputs are typical tyre performance characteristics in terms of both physical and micro-scale behaviour including vertical stiffness, Lateral stiffness, longitudinal stiffness, contact area, maximum contact pressure, maximum shear stress \#1 and maximum shear stress \#2 (direction \#1 refers to the direction of shear stress in lateral direction and direction \#2 corresponds with the longitudinal direction. The macro-scale parameters (stiffnesses) are normally specified design parameters while the micro-scale parameters (maximum contact pressure and shear stresses) must be kept below specified limits to ensure operational safety of the tyre.

\subsection{Implementation}

To facilitate the training of an artificial neural network, the available data is normally split into two sets: one set for training and another for testing. A limited number of training data set were randomly reserved for the purpose of cross validation, so that over fitting can be monitored and avoided. The trained neural networks can then be validated using the data set reserved for cross validation and the robustness of the ANN models assessed.

Table 4. Variation in input parameters of ANNs.

\begin{tabular}{cccc}
\hline Independent variables & low & normal & high \\
\hline Orientation angle & $-10 \%$ & $0 \%$ & $+10 \%$ \\
Cords end density & $-10 \%$ & $0 \%$ & $+10 \%$ \\
Material property & $-10 \%$ & $0 \%$ & $+10 \%$ \\
Cords X-section area & $-10 \%$ & $0 \%$ & $+10 \%$ \\
Inflation pressure & $-25 \%$ & $0 \%$ & $+25 \%$ \\
\hline
\end{tabular}

In this paper, the aforementioned neural networks were developed using the Matlab/NN toolbox in order to learn the same training data set. The network robustness was then examined using the same pre-selected set of test data for each of the networks. Test data were randomly chosen from $1 / 4$ to $1 / 3$ of the main data set. The performance and robustness of the different networks were compared so that the best configuration in terms of accuracy, performance and cost could be selected among available architectures.

The MLP was initially developed with single hidden layer, and the genetic algorithm approach was employed to optimise the number of neurons in the hidden layer in order to avoid manual searching for the optimum number of nodes in terms of accuracy. MLPs with more hidden layers were evaluated using the same training data. The correlation results did not show any improvement in accuracy, but the calculation cost increased as more hidden layers were added. The simulation was then extended to train and optimise RBF and Generalised MLP structures.

Genetic algorithm (GA) is an optimisation tool, which has its origin from evolution theory and Genetic science. Input parameters, initial population in GA terminology, are selected based on their calculated fitness index with an evaluation function, and the parent's pool is formed. Then the parent is mated (Mating, Crossover, and generating offspring) and a number of chromosomes are altered within the new generation randomly (Mutation). The new chromosomes make the next generation, which is then evaluated by fitness function again. The abovementioned steps are iterated until the appropriate fitness is met. The networks of this study were trained by GA where applicable (it was not applicable to CANFIS) and the number of neurons was calculated based on closer correlation of networks' output with desired values. Hence, the fitness function in GA is typically formulated such that network error would be the minimum at the end of training for test samples.

The back-propagation learning rule with an additional momentum term was selected for training MLPs and the other neural networks owing to its wide usage, low calculation cost, and good accuracy. However, a more accurate result can be achieved by using methods such as Levenberg-Marquardt learning algorithm [21]. Then the step and momentum size were kept constant in each individual hidden layer. In addition, the Tangent hyperbolic transfer function was used in hidden and output layers of the MLP architecture where applicable. The usage of transfer functions can be either optional or imperative according to the network's imposed architecture.

The CANFIS was finally developed and tested with the provided training and validation data. Bell-shape membership function was chosen along with Takagi-SugenoKang (TSK) model [22] as employed fuzzy model. In 
addition, three membership functions were set per input. The momentum played its role as the learning rule in this configuration as well.

In the developed network, the weights were updated by batch approach where applicable and the mean square error (MSE) method was selected as the performance index and error calculator. The training session for each network is kept running until the MSE error reaches its pre-defined error threshold of 0.01 . Then, the training session is labelled as completed. The GA optimisation is also regarded as finished after reaching 100 generations whilst 50 chromosomes are generated and selected in each generation.

\section{Results and Discussion}

The training sessions for four ANN structures were per-

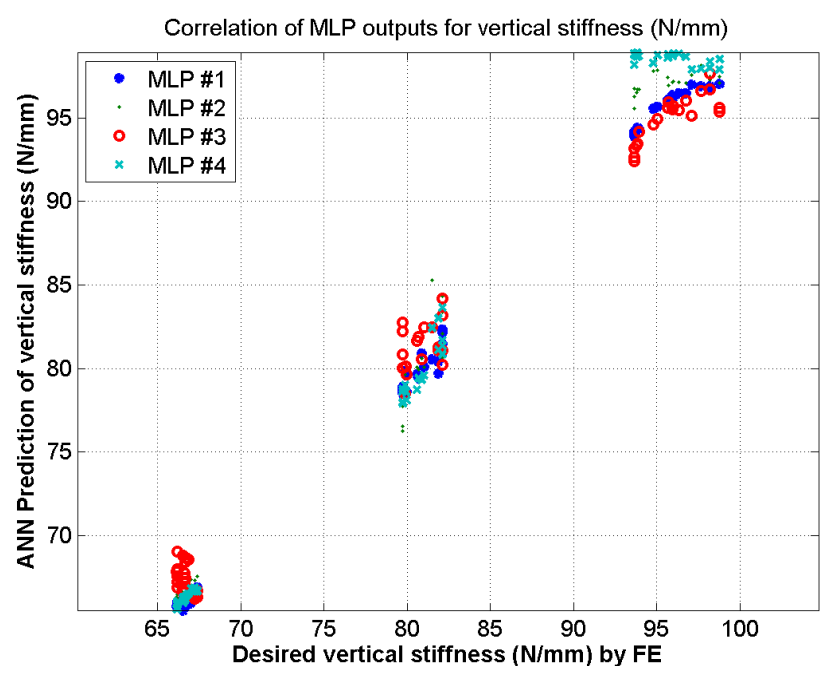

(a) formed by using three quarters of the sample pool randomly selected and the remaining one quarter was used for testing the developed networks. ANN output results have shown very accurate prediction of new samples. The results were compared in terms of their correlation factor, training effort, and network performance when encountering new data. Figures 5(a) and (b) show examples of comparison between results obtained from the trained ANN models using MLP structure and FE model. Each plot is formed of individual output results obtained from different MLP structures against the desired values extracted from FE model. Figure 5(a) illustrates the correlation of vertical stiffness while Figure 5(b) illustrates the correlation of contact area.

The correlation results for the different MLP structures are reported in Table 5. In these simulations, GA opti-

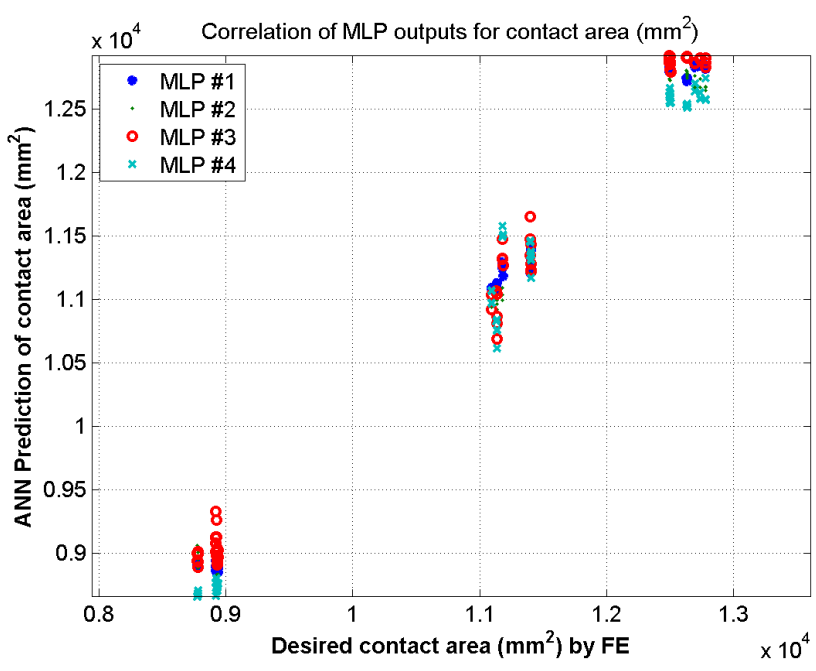

(b)

Figure 5. (a) A comparison between vertical stiffness of MLP outputs and virtual test results; (b) A comparison between contact area of MLP outputs and virtual test results.

Table 5. MLPs' configuration, training cost, and evaluated correlation factor between results by ANN and FE.

\begin{tabular}{|c|c|c|c|c|c|c|c|c|c|c|c|c|c|c|c|c|}
\hline Network type & 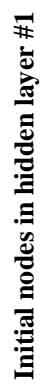 & 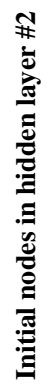 & 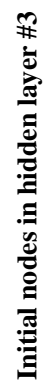 & 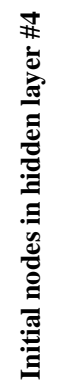 & 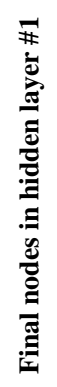 & 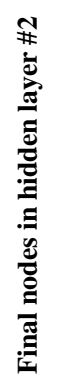 & 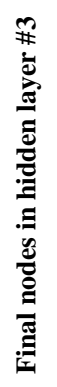 & 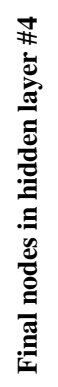 & 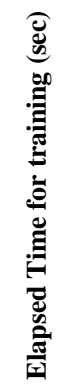 & 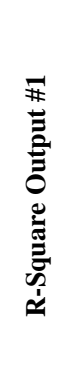 & 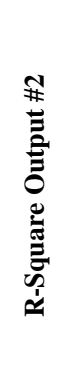 & 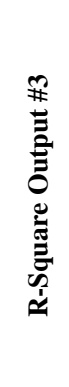 & 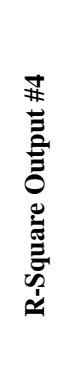 & 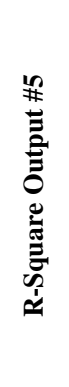 & 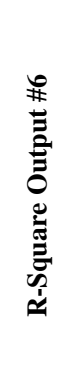 & 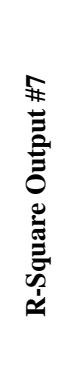 \\
\hline MLP with one hidden layer & 7 & 0 & 0 & 0 & 10 & 0 & 0 & 0 & 30 & 1 & 0.99 & 1 & 1 & 0.96 & 0.95 & 0.95 \\
\hline MLP with two hidden layers & 7 & 7 & 0 & 0 & 21 & 24 & 0 & 0 & 85 & 0.99 & 0.99 & 0.99 & 0.99 & 0.99 & 0.96 & 0.95 \\
\hline MLP with three hidden layers & 7 & 7 & 7 & 0 & 21 & 22 & 25 & 0 & 469 & 0.99 & 0.99 & 0.99 & 0.99 & 0.98 & 0.96 & 0.95 \\
\hline MLP with four hidden layers & 7 & 7 & 7 & 7 & 17 & 21 & 22 & 23 & 1922 & 0.99 & 0.99 & 0.99 & 0.99 & 0.97 & 0.95 & 0.96 \\
\hline
\end{tabular}


misation algorithm changed the number of neurons in hidden layer(s) to obtain an optimised structure in terms of accuracy and efficiency. Correlation factors are extremely close to one for macro-scale behaviour (stiffnesses and foot print area) and very close for micro-scale behaviour. The network efficiency in the training session was also evaluated by the elapsed time for training as shown in Table 5.

However, the ANN structures should also be evaluated for their online usage efficiency. The use of a smaller network structure (in terms of number of layers and nodes) requires a smaller number of main mathematical operations (number of main mathematical operations is the major source of calculation cost of formulas). It will consequently lead to faster evaluation of ANN when a network with less complexity and fewer hidden layers is employed.

The time needed for training session increases exponentially as the network complexity is increased by employing more hidden layers and GA implementation on the network will severely influence the processing time for the training session. There is, nevertheless, an exception when the CANFIS is being trained. The elapsed time for training is substantially higher for a two-layer configuration because of the high cost of optimising three membership functions morphology to achieve the best fitness.

Figure 6 shows a comparison of the training efficiency of the different networks. GFMLP showed the lowest elapsed time for training in spite of its more complex structure in comparison with MLP while CANFIS achieved the poorest performance in training. The correlation factor, $R^{2}$, achieved by the trained ANN network configurations in each of the seven desired outputs in comparison with FE simulation results is shown in Figure 7. Figure 8 shows the average correlation factors achieved over the seven desired outputs for the trained ANN network configurations. Most of the ANN configurations have shown reliable and acceptable results for the randomly selected test data. MLP outputs have achieved the best correlation with FE results even for the simplest configuration of one hidden layer. The training session cost for GFMLP is the least expensive of all the configurations as can be seen from Figure 6. MLPs are also quite efficient, matching GFMLPs in terms of cost of training particularly for the first two configurations (MLP \#1-2), but not for higher number of hidden layers.

Hence overall, it has been demonstrated that the MLPs can be deployed with adequate efficiency as well as outstanding accuracy for the set of available data in this research. MLP with two hidden layers produced the best combination of accuracy and low cost of training. Moreover, MLP is the simplest and most commonly used structure among the proposed network structures.

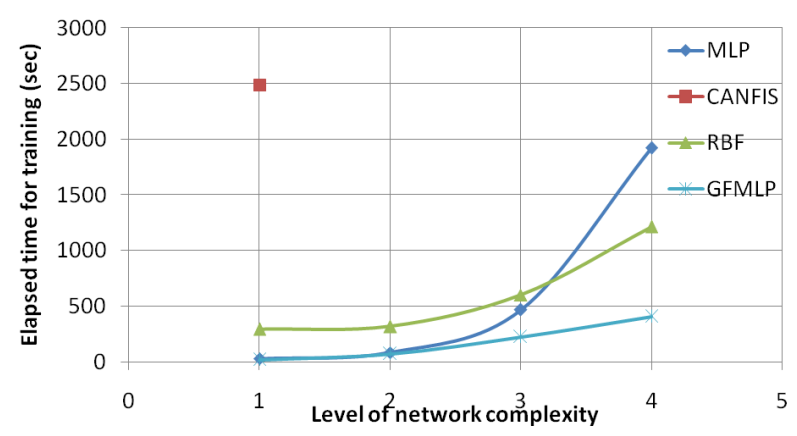

Figure 6. Efficiency of ANNs in training session.

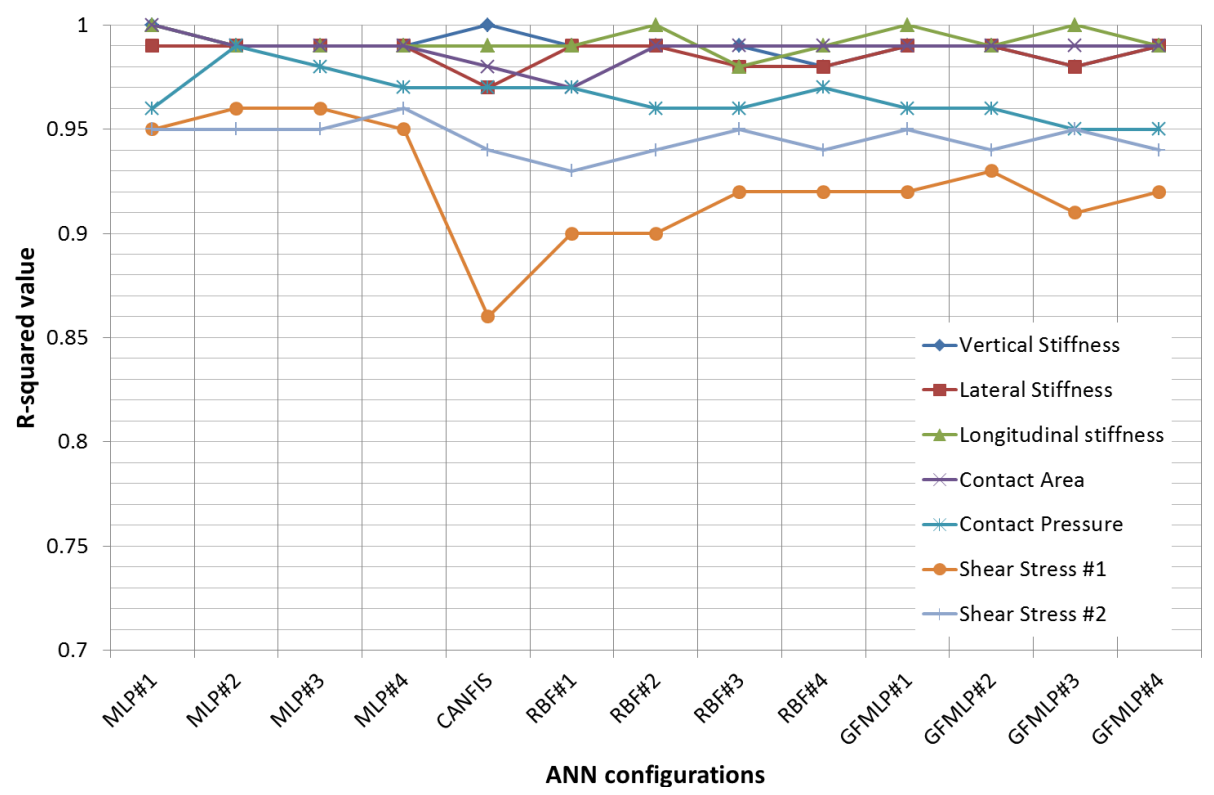

Figure 7. Comparison of $\mathbf{R}^{2}$ values of desired outputs between ANN prediction and FE results. 


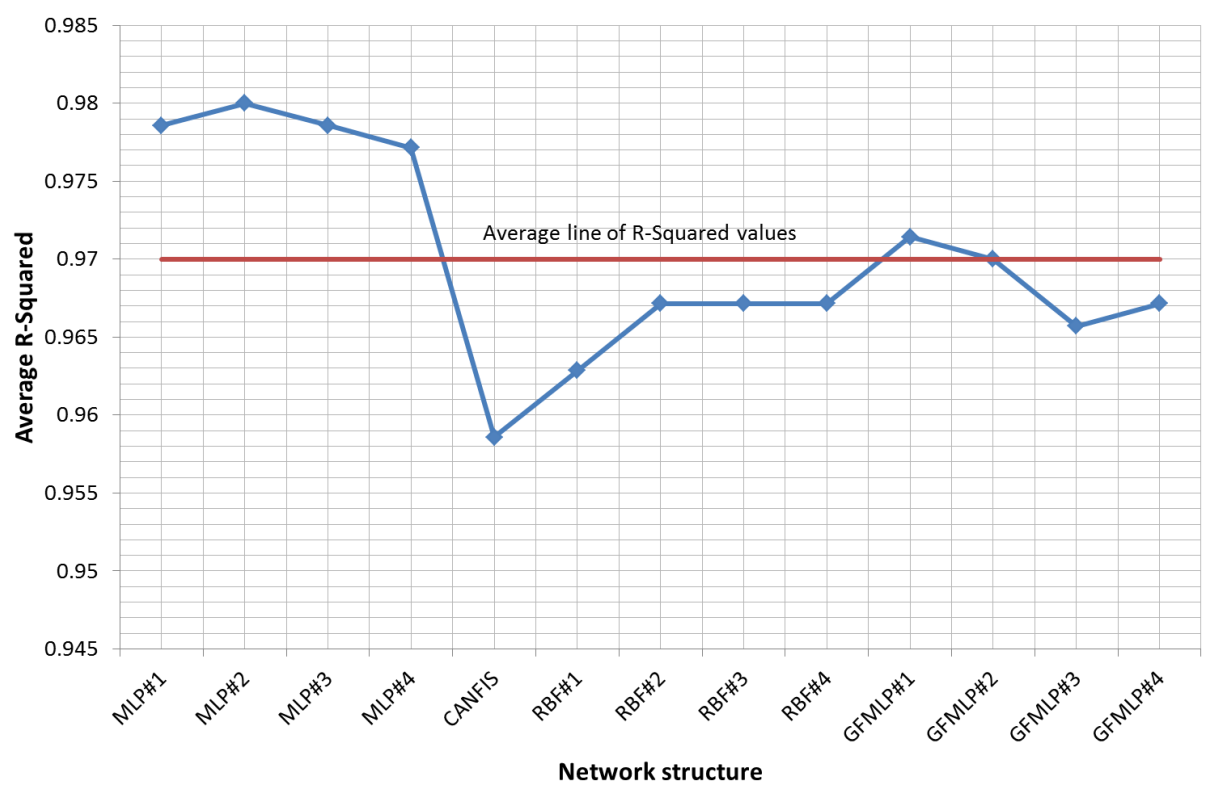

Figure 8. Comparison of average $\mathbf{R}^{2}$ values of desired outputs between ANN prediction and FE results.

\section{Conclusions}

In this study, an FE tyre model was developed to simulate tyre behaviour. Validation of the FE model was performed in order to confirm whether simulated results were matched properly to real-world tests. Then, virtual tests were developed to generate tyre behaviour characteristics under various tyre designs and loading configurations using the validated tyre model. Next, these results, along with the input data, were used in modelling an ANN-based system to predict tyre characteristics. Various ANN configurations were developed using Matlab/NN Toolbox to learn the complex relationships between the geometrical and load parameters as input data and tyre stiffness properties and stresses as outputs. The network efficiency and performance of the different configurations were compared by using a randomly selected new dataset. The MLP and GFMLP configurations exhibited the best combination of accuracy and training efficiency out of all the configurations for the provided tyre training data set. The MLP with two hidden layers exhibited the best overall performance.

This ANN configuration can predict tyre performance characteristics such as vertical and lateral stiffnesses, contact pressure etc. for new tyre designs. The significance of this is that such an ANN can enable extensive parametric studies to be carried out, inexpensively, to optimise a new tyre design to achieve the design targets before a much more expensive full $\mathrm{FE}$ analysis is used to confirm the predicted performance. Prototypes can then be built and tested to confirm the predicted performance of the design. This can certainly help tyre designers to achieve an optimised design quickly, efficiently and at reduced cost.
This opens up the possibility of applying ANN to predicting other tyre performance characteristics as well as other automotive systems as an alternative to more expensive analysis methods. Possible areas of application, which will be explored in the future, are computing tyre burst inflation pressure, rolling resistance properties and tyre force and moment generation for simulating vehicle dynamics responses.

\section{REFERENCES}

[1] P. Gruber, R. S. Sharp and A. D. Crocombe, "Friction and Camber Influences on the Static Stiffness Properties of a Racing Tyre," Proceedings of the Institution of Mechanical Engineers, Part D: Journal of Automobile Engineering, Vol. 222, No. 11, 2008, pp. 1965-1976. http://dx.doi.org/10.1243/09544070JAUTO872

[2] K. Kabe and M. Koishi, "Tyre Cornering Simulation Using Finite Element Analysis,” Journal of Applied Polymer Science, Vol. 78, No. 8, 2000, pp. 1566-1572.

[3] O. A. Olatunbosun and A. M. Burke, "Finite Element Modelling of Rotating Tyres in the Time Domain," Tire Science and Technology, TSTCA, Vol. 30, No. 1, 2002, pp. 19-33.

[4] P. Ghosh, A. Saha and R. Mukhopadhyay, "Optimization of Tyre Design Parameters through Finite Element Analysis and Correlation with Performance,” 2007, SAE Paper No. 2007-26-043.

[5] J. Zhang, "Developing Robust Non-Linear Models through Bootstrap Aggregated Neural Networks," Neurocomputing, Vol. 25, No. 1-3, 1999, pp. 93-113. http://dx.doi.org/10.1016/S0925-2312(99)00054-5

[6] R. J. Patton and J. Chen, "Neural Networks Based Fault Diagnosis for Nonlinear Dynamic Systems,” AIAA Guidance, Navigation and Control Conference, Baltimore, 
7-10 August 1995, pp. 419-428.

[7] Y. H. Liu and L. L. Liu, "Sales Forecasting through Fuzzy Neural Networks,” International Conference on Electronic Computer Technology, Macau, 20-22 Febuary 2009, pp. 511-515.

[8] V. Ivanov, B. Shyrokau, K. Augsburg and V. Algin, "Fuzzy Applications to Tyre Environment," FISITA, 2008, F2008-12-001.

[9] J. Matusko, I. Petrovic and N. Peric, "Neural Network Based Tyre/Road Friction Force Estimation,” Engineering Applications of Artificial Intelligence, Vol. 21, No. 3, 2008, pp. 442-456.

[10] A. J. Barber, T. E. Renner, S. You, G. S. Sandlass and A. Maki, "Predicting Tyre Handling Performance Using Neural Network Models,” 2004, SAE Paper No. 2004-011574.

[11] A. Z. Al-Garn and A. Jamal, “Artificial Neural Network Application of Modelling Failure Rate for Boeing 737 Tyres," Applied Simulation and Modelling Conference, Corfu, 23-25 June 2008.

[12] O. A. Olatunbosun, X. Yang, M. Behroozi and D. GarciaPozuelo, "Development of an AI-Based Model to Determine Vehicle Tire Design Configuration,” FISITA World Congress, Budapest, 2010, Paper F2010-C-213.

[13] P. Clarke, “Tyres,” 2006. http://www.formulastudent.de/academy/pats-corner/advic e-details/article/tyres/

[14] “An Integrated Approach for Transient Rolling of Tyres," Abaqus Technology Brief, 2007.
[15] X. Yang, O. A. Olatunbosun and E. O. Bolarinwa, "Materials Testing for Finite Element Tyre Model," SAE International Journal of Materials and Manufacturing, Vol. 3, No. 1, 2010, pp. 211-220.

[16] E. O. Bolarinwa, "Investigation of the Dynamic Characteristics of Radial Tyre Using the Finite Element Method," Ph.D. Thesis, Mechanical Engineering, University of Birmingham, UK, 2004.

[17] S. Samarasinghe, "Neural Networks for Applied Sciences and Engineering: From Fundamentals to Complex Pattern Recognition,” 1st Edition, Auerbach Publications, 2006.

[18] E. Mizutani, “Coactive Neural Fuzzy Modelling,” Proceedings of IEEE International Conference on Neural Networks, Vol. 2, 1995, pp. 760-765. http://dx.doi.org/10.1109/ICNN.1995.487513

[19] P. V. Yee and S. Haykin, "Regularized Radial Basis Function Networks: Theory and Applications,” John Wiley, 2001.

[20] K. Hornik, M. Stinchombe and H. White, "Multilayer Feed-Forward Networks Are Universal Approximators," Neural Networks, Vol. 2, No. 5, 1989, pp. 359-366. http://dx.doi.org/10.1016/0893-6080(89)90020-8

[21] S. Haykin, "Neural Networks: A Comprehensive Foundation,” 2nd Edition, Prentice Hall PTR, Upper Saddle River, 1998.

[22] M. Sugeno and G. Kang, "Structure Identification of Fuzzy Model,” Fuzzy Sets and Systems, Vol. 28, No. 3, 1986, pp. 329-346. http://dx.doi.org/10.1016/0165-0114(86)90010-2 